\title{
TANGENTS OF $\sigma$-FINITE CURVES AND SCALED OSCILLATION
}

\author{
MARIANNA CSÖRNYEI AND BOBBY WILSON
}

\begin{abstract}
Aвstract. We show that every continuous simple curve with $\sigma$-finite length has a tangent at positively many points. We also apply this result to functions with finite lower scaled oscillation; and study the validity of the results in higher dimension.
\end{abstract}

\section{INTRODUCTION}

Consider a continuous function $f: \Omega \subset \mathbb{R}^{n} \rightarrow \mathbb{R}$. We begin with the well-known notion of Lipschitz continuity and Rademacher's Theorem which states that if $f$ is Lipschitz continuous on the domain $\Omega$, then $f$ is differentiable almost everywhere on $\Omega$. A generalization of Rademacher's Theorem, known as Stepanov's theorem, states that the conclusion of Rademacher's theorem holds under the assumption that the upper scaled oscillation, (see Def. 4), is finite for almost every point in $\Omega$.

A natural question to ask is whether or not the assumptions of Stepanov's theorem can be reduced to assuming the lower scaled oscillation is finite for almost all $x \in \Omega$ while preserving the conclusions. Balogh and Csörnyei [2] proved that one cannot simply replace upper scaled oscillation with lower scaled oscillation and acquire the same results. Indeed, for $n=1$, they constructed a nowhere differentiable, continuous function $f:[0,1] \rightarrow \mathbb{R}$ such that $l_{f}(x)=0$ for a.e. $x \in$ $[0,1]$. However, they were able to show that if the lower scaled oscillation is finite on all but countably many points of $[0,1]$, then $f$ is always differentiable on a set of positive measure, though it is not necessarily differentiable almost everywhere.

The results of our paper were initiated by the observation (see Lemma 11) that the graph of such $f$ has $\sigma$-finite $\mathcal{H}^{1}$ measure and $f$ satisfies Luzin's $(N)$ condition. This naturally leads to the question whether every curve of $\sigma$-finite length has a tangent at every point of a set of positive $\mathcal{H}^{1}$ measure.

It is well-known that every curve of finite length has a tangent at a.e. of its points. Therefore the answer to this question is positive when the curve can be decomposed into countably many sub-arcs, each of which has finite length. However, there are curves of $\sigma$-finite $\mathcal{H}^{1}$ measure whose every sub-arc is of infinite length.

Every set $E$ of $\sigma$-finite $\mathcal{H}^{1}$ measure can be decomposed into a rectifiable and a purely unrectifiable part. The tangent of a rectifiable set is well-understood, for a detailed discussion see e.g. Chapter 15 in [7]. The purely unrectifiable part has zero projection in a.e. direction, therefore, if $E$ is a curve, then its rectifiable part cannot be negligible, so the tangent of $E$ is defined at positively many points. Besicovitch discusses the special case when the set is a simple curve in [3] and [4]. The definition of tangent was later generalized to an arbitrary Lebesgue null set in [1].

2010 Mathematics Subject Classification. 26A24, 28A15.

Key words and phrases. Differentiability, Rademacher's Theorem, Lipschitz, Scaled Oscillation, Luzin's condition, $\sigma$-finite Curves. 
Both the standard definition of the tangents of rectifiable sets and the definition in [1] for Lebesgue null sets are given in the "almost everywhere" sense: if we change the tangent field on a sufficiently small set (namely, on a purely unrectifiable set), it is still a tangent field. Therefore these definitions cannot say what the tangent of the set is at a given point. In [4], Besicovitch showed that there exists a continuous function defined on $[0,1]$ with a $\sigma$-finite graph and for which there exists a set $E \subset[0,1]$, with $\mathcal{L}^{1}(E)=\frac{1}{2}$, such that $f$ is not differentiable on $E$. He also asserted with his demonstration of this example that "...it is hardly possible to find a satisfactory definition of a tangent to a curve of $\sigma$-finite length at a point considered individually."

However, in this paper we show that every $\sigma$-finite curve has a tangent, in the pointwise sense, on a set of positive measure. By "tangent" we mean the classical notion: the limit direction from which the curve approaches the point (see Section 2). We prove the following theorem:

Theorem 1. Let $C$ be a closed, continuous simple curve in $\mathbb{R}^{n}$. Assume that $C$ has $\sigma$-finite $\mathcal{H}^{1}$ measure. Then $C$ has a tangent at positively many of its points.

We also show how this theorem can be used to give a very quick proof of the result of [2] in dimension $n=1$ mentioned above, and we also extend it to higher dimension.

In [2], it was proved that a function $f:[0,1]^{n} \rightarrow \mathbb{R}$ is differentiable almost everywhere, provided that $l_{f}(x)<\infty$ except at the points of a set $E$ with $\sigma$-finite $\mathcal{H}^{n-1}$ measure, and $l_{f} \in L^{p}$ where $p>n$. Hanson showed in [6] that one cannot weaken the hypotheses that $E$ has $\sigma$-finite $(n-1)$-dimensional measure: he gave a sharp estimate for the gauge functions $g$ for which the theorem remains true with $\mathcal{H}^{n-1}$ replaced with $\mathcal{H}^{8}$. In particular, he showed that $E$ can have Hausdorff dimension at most $n-1$.

In view of the positive results for $n=1$ mentioned at the beginning of this introduction, it is natural to conjecture that, though a function $f:[0,1]^{n} \rightarrow \mathbb{R}$ may not be differentiable almost everywhere, it is still differentiable on a set of positive measure, provided that $l_{f}(x)<\infty$ outside a set of $\sigma$-finite $\mathcal{H}^{n-1}$ measure. Here we do not assume that $l_{f} \in L^{p}$.

Surprisingly, we show that this conjecture is false for $n>1$. Theorem 1 holds true only in dimension one, higher dimensional $\sigma$-finite surfaces do not necessarily have tangents at any points. Through this observation we can construct nondifferentiable functions for any gauge function $g$. We prove the following theorem:

Theorem 2. For any $n \geq 1$ and for any non-zero gauge function $g$ there exists an almost everywhere non-differentiable continuous function $f:[0,1]^{n} \rightarrow \mathbb{R}$ with $l_{f}(x)<\infty$ at all points in $[0,1]^{n}$ except on a set $E$ with $\mathcal{H}^{g}(E)=0$.

However, for $\mathcal{H}^{0}$, we obtain a positive result in any dimension. This answers a question of Hanson.

Theorem 3. Let $f:[0,1]^{n} \rightarrow \mathbb{R}$ be a continuous function. Assume $l_{f}(x)<\infty$ for all but at most countably many $x \in[0,1]^{n}$. Then $f$ is differentiable at the points of a set of positive measure.

Geometrically, the main difference between an exceptional set of $\sigma$-finite $\mathcal{H}^{n-1}$ (or, $\mathcal{H}^{g}$ ) measure and a countable exceptional set is that the former one may be mapped onto a large set by a continuous function, while, no matter what, the image 
of a countable set is always countable. Although, the validity of Theorem 3 was an open problem, even when assuming the exceptional set is empty.

\section{Preliminaries}

To start with, we fix some notations. As usual, we let $\mathcal{L}^{n}$ denote the $n$ dimensional Lebesgue measure and $\mathcal{H}^{s}$ denotes the s-dimensional Hausdorff measure for $n \in \mathbb{N}$ and $s \in[0, \infty)$. We will also use Hausdorff measures $\mathcal{H}^{g}$ where $g$ is a gauge function. Recall that a function $g:[0, \infty) \rightarrow[0, \infty)$ is called a gauge function, if it is continuous, monotone increasing, $g(0)=0$ and $g(t)>0$ for $t>0$. The Hausdorff measure $\mathcal{H}^{g}$ is defined as the limit, $\lim _{\delta \rightarrow 0} \mathcal{H}_{\delta}^{g}$, where for a given set E,

$$
\mathcal{H}_{\delta}^{g}(E)=\inf \left\{\sum_{i} g\left(\operatorname{diam}\left(E_{i}\right)\right) \mid E \subset \bigcup_{i} E_{i}, \operatorname{diam}\left(E_{i}\right)<\delta\right\} .
$$

We denote the open ball around $x$ of radius $r$ by $B(x, r)$, and, for any direction $\theta$, the orthogonal projection onto $\theta^{\perp}$ by $\operatorname{proj}_{\theta}$.

With respect to inequalities, we will commonly use $a \lesssim b$ to denote instances when there exists a constant $C \in(0, \infty)$ such that $a \leq C b$ and $a \ll b$ in situations where $a<C b$ for some positive constant $C$ much smaller than 1 .

We define:

Definition 4 (Upper/Lower Scaled Oscillation). Let $(X, d)$ be a metric space, $f: X \rightarrow$ $\mathbb{R}^{n}$ be continuous. For $x \in X$ define the upper and lower scaled oscillation functions of $f$ as:

$$
\begin{aligned}
L_{f}(x) & :=\limsup _{r \rightarrow 0} \frac{\sup _{d(x, y) \leq r}\|f(y)-f(x)\|}{r} \\
l_{f}(x) & :=\liminf _{r \rightarrow 0} \frac{\sup _{d(x, y) \leq r}\|f(y)-f(x)\|}{r}
\end{aligned}
$$

Next, we state some definitions and a theorem that appear in [9]. The half-line in $\mathbb{R}^{n}$ issuing from a point $x$ and containing a point $y \neq x$ will be denoted by $x \vec{y}$. Given $E \subset \mathbb{R}^{n}$, a half-line $L$ issuing from a point $x \in E$ is called an intermediate half-tangent of $E$ at $x$, if there exists a sequence $\left\{y_{n}\right\}$ of points of $E$ distinct from $x$, converging to $x$ and such that the sequence of half-lines $\left\{x \vec{y}_{n}\right\}$ converges to $L$.

Definition 5 (Contingent). The set of all intermediate half-tangents of $a$ set $E$ at a point $x$ is termed the contingent of $E$ at $x$ and denoted by $\operatorname{contg}_{E} x$.

When $E \subset \mathbb{R}^{n}$ is homeomorphic to $[0,1]^{k}$, and the contingent of $E$, at a point $x$, is a $k$-dimensional plane, then we say that it is the tangent of $E$ at $x$. Indeed, geometrically, this means that locally around $x$ the set $E$ can be very well approximated by this $k$-plane: in a small enough neighborhood of $x$, all the half-lines $\overrightarrow{x y}$ are within a very small angle of the plane. In addition, close to any direction in the plane, there is an $\overrightarrow{x y}$.

In the special case when $E$ is the graph of a continuous function $f$, the function $f$ is differentiable at $x$ if and only if its graph has a tangent at the point $(x, f(x))$ and this tangent is not a vertical plane (i.e. it does not contain the vertical line). Also note that, for any function $f$, at least one of the two vertical half-lines belongs to the contingent of the graph of the function at $(x, f(x))$ if and only if $L_{f}(x)=\infty$. On the 
other hand, $l_{f}(x)<\infty$ implies that the contingent contains at least one non-vertical half-line.

We will use the following lemma from Saks [9]:

Lemma 6 (Theorem 3.6, Chapter IX in Saks [9]). Given a planar set $R$, let $P$ be a subset of $R$ at no point of which the contingent of $R$ is the whole plane. Then

i) the set $P$ is a countable union of sets of finite length and

ii) at every point of $P$, except at those of a set of length zero, either the set $R$ has a tangent or else the contingent of $R$ is a half-plane.

The analogous result can also be proved for sets in $\mathbb{R}^{n}$ for any $n$ by the same argument as the planar proof in [9] (see [8]). At each point except at those of a set of zero $(n-1)$-dimensional measure, the contingent is either the whole space, or a halfspace, or an ( $n-1)$-plane. In this paper, in dimensions $n \geq 3$, we will use only the following corollary, that can also be thought of as a variant of Stepanov's theorem mentioned in the introduction:

Corollary 7. Let $f: \mathbb{R}^{n} \rightarrow \mathbb{R}$ be a continuous function. Then $L_{f}(x)=\infty$ at almost every point $x$ where $f$ is non-differentiable.

\section{3. $\sigma$-FINITE CURVES AND DIFFERENTIABILITY}

In what follows, we will use the notation $F_{x}^{\theta}$ for the $(n-1)$-dimensional plane through the point $x \in \mathbb{R}^{n}$ of normal direction $\theta$.

First, let $C$ be a continuous simple curve in the plane. We show that we have control of the contingent for points that are in some sense isolated with respect to the curve:

Lemma 8. Let $C$ be a continuous, closed simple curve in the plane and suppose that on a set $D \subset C$, there exist three directions $\theta_{1}, \theta_{2}, \theta_{3}$ such that for every $z \in D$, $z$ is isolated in $C \cap F_{z}^{\theta_{i}}$ for each $i \in\{1,2,3\}$. Then at almost all $z \in D, C$ has a tangent line.

Proof. For a fixed $z \in D$, there exists a $\delta$-ball around $z$ such that $F_{z}^{\theta_{i}} \cap C \cap B(z, \delta)=\{z\}$ for each $i$. The $F_{z}^{\theta_{i}}$ decompose the $\delta$-ball around $z$ into 6 regions. The curve is simple and continuous, so the curve can only lie in at most 2 of the 6 regions around $z$. Therefore the contingent of $C$ cannot be the whole plane or a halfplane. By Lemma 6. at all points of $D$ except at those of a set of length zero, $C$ has a tangent.

Remark 9. We observe that it suffices to assume that the curve is injective at almost every point of $D$ to prove Lemma 8 Any self-intersections away from points in $D$ will not affect the argument.

In the planar case we prove Theorem 1 by showing that if $C$ has $\sigma$-finite $\mathcal{H}^{1}$ measure then there exists a set $D$ of positive $\mathcal{H}^{1}$ measure for which Lemma 8 can be applied. This is a corollary of a theorem from Falconer [5].

Lemma 10 (Falconer [5], Corollary to Theorem 5.8). Consider $E \subset \mathbb{R}^{d}$ and let $A$ be a subset of a primary axis. Let $E_{x}=F_{x}^{\theta} \cap E$, where $\theta$ is the direction of the primary axis. Suppose that if $x \in A$, then $\mathcal{H}^{0}\left(E_{x}\right)>c$, for some constant $c$. Then

$$
\mathcal{H}^{s}(E) \geq b c \mathcal{H}^{s}(A)
$$

where $b$ depends on $s$ and $d$. 
Proof of Theorem 1 in the plane. Let $\theta \in(0, \pi)$ be some angle and $L_{\theta}$ be the line with angle $\theta$ through the origin. We first note that

$$
\mathcal{H}^{1}\left[\operatorname{proj}_{\theta}(C)\right]>0
$$

for all but at most one $\theta$. Therefore, without loss of generality we assume $\mathcal{H}^{1}\left[\operatorname{proj}_{\theta}(C)\right]>0$. Consider the decomposition of $C,\left\{C_{M}\right\}$, given by the $\sigma$-finite condition, and denote $E_{M}:=\bigcup_{1}^{M} C_{i}$. Since

$$
\operatorname{proj}_{\theta}\left(E_{M}\right) \stackrel{M \rightarrow \infty}{\longrightarrow} \operatorname{proj}_{\theta}(C),
$$

there exists an $M_{0}$ such that $\mathcal{H}^{1}\left[\operatorname{proj}_{\theta}\left(E_{M}\right)\right]>0$ for $M \geq M_{0}$. Now

$$
0<\mathcal{H}^{1}\left[\operatorname{proj}_{\theta}\left(E_{M_{0}}\right)\right] \leq \mathcal{H}^{1}\left(E_{M_{0}}\right)<\infty .
$$

By Lemma 10 .

$$
\mathcal{H}^{0}\left[E_{M_{0}} \cap F_{z}^{\theta}\right]<\infty
$$

for almost all $z \in \operatorname{proj}_{\theta}\left(E_{M_{0}}\right)$. Furthermore, for almost all $z \in \operatorname{proj}_{\theta}\left(E_{M_{0}}\right)$,

$$
\mathcal{H}^{0}\left[E_{M} \cap F_{z}^{\theta}\right]<\infty
$$

for $M>M_{0}$. Thus, $C \cap F_{z}^{\theta}$ is at most countable for almost all $z \in \operatorname{proj}_{\theta}\left(E_{M_{0}}\right)$.

Every closed countable set of points must contain an isolated point. Let $X_{\theta}$ be the set of points in $C$ that are isolated in $C \cap F_{z}^{\theta}$ for some $z$. Then $X_{\theta}$ contains at least one point of $C \cap F_{z}^{\theta}$ for almost all $z \in \operatorname{proj}_{\theta}\left(E_{M_{0}}\right)$.

In order to show that $X_{\theta}$ is a Borel set, we can show that its complement is an $F_{\sigma \delta}$ set. Consider, for each $n, m \in \mathbb{N}, n>m$, the set

$$
F_{n, m}:=\left\{x \in C \mid \text { There exists a } y \in C \cap F_{x}^{\theta} \text { such that }\|x-y\| \in\left[\frac{1}{n}, \frac{1}{m}\right]\right\} .
$$

Since $C$ is closed $F_{n, m}$ is closed. We take the union over $n$ and then the intersection over $m$ and we obtain the complement to $X_{\theta}$.

By Lemma 10, $\mathcal{H}^{1}\left(X_{\theta}\right)>0$. This holds true for uncountably many $\theta$. Therefore, there exist 3 angles $\theta_{1}, \theta_{2}, \theta_{3}$ such that

$$
\mathcal{H}^{1}\left(\bigcap_{i} X_{\theta_{i}}\right)>0
$$

and we can apply Lemma 8 with $D=\bigcap_{i} X_{\theta_{i}}$ to obtain positively many points at which $C$ has a tangent.

Proof of Theorem 1 in higher dimension. We show that our arguments can be extended to simple curves in $\mathbb{R}^{n}$. Let us first consider the case $n=3$.

For any direction $\theta \in S^{2}$, if $\operatorname{proj}_{\theta} C$ has a tangent $\tau$ at $\operatorname{proj}_{\theta} x$, then $\operatorname{contg}_{C} x$ must lie inside the 2-dimensional plane $\operatorname{proj}_{\theta}^{-1} \tau$. For almost all directions, almost all 2 dimensional plane sections will intersect the curve at isolated points (with respect to the section) for positively many points of the curve. By Lemma 8 and Remark 9. it is sufficient to find 6 directions $\eta_{1}, \eta_{2}, \eta_{3}$ and $\eta_{4}, \eta_{5}, \eta_{6}$ : two triples belonging to two different planes $\theta_{1}^{\perp}, \theta_{2}^{\perp}$, such that each point $x$ in a subset $D \subset C$ is isolated in each of the six 2-dimensional sections $F_{x}^{\eta_{j}} \cap C$, and for which $D$ has positive measure. Then for each $i=1,2$ and for a sufficiently small $\delta$, we can find tangent 
$\tau_{i}$ of $\operatorname{proj}_{\theta_{i}}(C \cap B(x, \delta))$ at $\operatorname{proj}_{\theta_{i}} x$ at positively many $x \in D$, and the tangent of $C$ at $x$ will be the line $\bigcap_{i} \operatorname{proj}_{\theta_{i}}^{-1} \tau_{i}$.

The higher dimensions follow by taking more planes.

Now, let $f: \mathbb{R}^{n} \rightarrow \mathbb{R}^{m}$ be a continuous mapping, and let $G_{f}(E)$ denote the graph of $f$ on a set $E \subset \mathbb{R}^{n}$. The following lemma shows that the finite lower scaled oscillation implies a certain $\sigma$-finite condition and a Luzin type condition. Actually, we will use this lemma only in the special case when $m=s=1$ and $E$ is a simple curve of finite length. However, the validity of this lemma in higher dimensions is the main motivation behind our construction of a surface $S$ in Section 4 that we will use in the proof of Theorem 2 So let us state our lemma in its full generality:

Lemma 11. Let $s>0$ be arbitrary, let $E \subset \mathbb{R}^{n}$ be a Borel set, and let $f: E \rightarrow \mathbb{R}^{m}$ be a continuous mapping satisfying $l_{f}(x)<\infty$ and $\liminf _{r \rightarrow 0} \mathcal{H}^{s}(E \cap B(x, r)) / r^{s}>0$ at all but countably many points $x \in E$. Then

(1) $G_{f}(E)$ has $\sigma$-finite $\mathcal{H}^{s}$-measure.

(2) $\mathcal{H}^{s}\left[G_{f}(H)\right]=0$ for every set $H \subset E$ with $\mathcal{H}^{s}(H)=0$.

Note that in the special case when $E$ is a continuous curve and $s=1$, the condition $\lim _{\inf _{r \rightarrow 0}} \mathcal{H}^{1}(E \cap B(x, r)) / r>0$ is automatically satisfied. However, this is not true for $k$-dimensional surfaces and $\mathcal{H}^{k}$.

Proof. The image of the countable exceptional set is countable, therefore it is enough to prove that the graph above the non-exceptional points have $\sigma$-finite $\mathcal{H}^{s}$ measure and that this part of the graph satisfies Luzin condition (2).

For given positive numbers $M$ and $t$, denote

$$
F=E_{M, t}:=\left\{x \in E \mid l_{f}(x)<M, \liminf _{r \rightarrow 0} \mathcal{H}^{s}(E \cap B(x, r)) / r^{s}>t\right\} .
$$

For part (1), it is sufficient to prove that $\mathcal{H}^{s}\left(G_{f}(F)\right)<\infty$ for any $M$ and $t$.

The hypothesis implies that for an arbitrary small $\delta$ there exists a covering of $F$ by balls of diameter less than $\delta$, such that for each ball $B=B(x, r),\|f(x)-f(y)\| \leq M r$ for all $y \in B$, and $\mathcal{H}^{s}(E \cap B) \geq t r^{s}$.

Now let $\left\{B_{i}\right\}$ (with centers $x_{i}$ and radii $r_{i}$ ) be a subcover given by the Besicovitch Covering Theorem. We note that $\left\|f\left(x_{i}\right)-f(y)\right\| \leq M r_{i}$ for every $y \in B_{i}$. Then, with $c_{M}=\sqrt{M^{2}+1}$, the balls around the points $\left(x_{i}, f\left(x_{i}\right)\right)$ of diameter $c_{M} \operatorname{diam}\left(B_{i}\right)<c_{M} \delta$ cover $G_{f}(F)$, therefore

$$
\begin{aligned}
\mathcal{H}_{c_{M} \delta}^{\varsigma}\left(G_{f}(F)\right) & \leq \sum_{i}\left(2 c_{M} \operatorname{diam}\left(B_{i}\right)\right)^{s} \leq c_{M, s, t} \sum_{i} \mathcal{H}^{\varsigma}\left(E \cap B_{i}\right) \leq \\
& \leq c_{n, M, s, t} \mathcal{H}^{s}(E)<\infty .
\end{aligned}
$$

For part (2), consider $H_{M, t}:=H \cap E_{M, t}$, and $\epsilon>0$ arbitrary, and cover $H_{M, t}$ by a relatively open set $U \subset E$ with $\mathcal{H}^{s}(U)<\epsilon / c_{n, M, s, t}$. The hypothesis of our lemma remains true with $E$ replaced by $U$, therefore our argument above shows that $\mathcal{H}^{s}\left(G_{f}\left(H_{M, t}\right)\right) \leq \mathcal{H}^{s}\left(G_{f}\left(U_{M, t}\right)\right) \leq c_{n, M, s, t} \mathcal{H}^{s}(U)<\epsilon$. This holds for any $\epsilon$, therefore $\mathcal{H}^{s}\left(G_{f}\left(H_{M, t}\right)\right)=0$.

Theorem 1 combined with Lemma 11 gives an alternative proof to the positive result in [2] for $n=1$ mentioned in the beginning of the introduction. Moreover:

Corollary 12. Let $\gamma \subset[0,1]^{n}$ be a simple curve with finite length, and let $f: \gamma \rightarrow \mathbb{R}$ be a continuous function. Assume $l_{f}(x)<\infty$ for all but at most countably many $x$ in $\gamma$. Then $f$ is differentiable at positively many points of $\gamma$. 
Indeed, applying part (1) of Lemma 11 with $E=\gamma$ and $m=s=1$, we can see that $G_{f}(E)$ is a simple curve in $\mathbb{R}^{n+1}$ of $\sigma$-finite length. By Theorem 1 , it has a tangent at a subset $D$ with $\mathcal{H}^{1}(D)>0$. By part (2), this set $D$ lies above a positive subset of $\gamma$. In order to prove that $f$ is differentiable at these points, the only thing we need to check is that the tangent line is not vertical. This follows immediately from the property $l_{f}(x)<\infty$.

We now show how Corollary 12 can be used to prove Theorem 3 . The key step in our proof is the following lemma:

Lemma 13. Let $f:[0,1]^{n} \rightarrow \mathbb{R}$ be a continuous function, and assume $L_{f}(x)=\infty$ at almost every $x \in[0,1]^{n}$. Then there exists a simple curve $\gamma \subset[0,1]^{n}$ of finite length, such that $L_{g}(x)=\infty$ for $g:=\left.f\right|_{\gamma}$ and for $\mathcal{H}^{1}$-a.e. $x$ in $\gamma$.

Assume that Theorem 3 is false for some function $f$. Then, by Corollary 7 , it satisfies the hypotheses of Lemma 13 . This gives us a curve $\gamma$ with the property that for almost all $x$ in $\gamma$, the contingent of the graph of $g$ at $(x, g(x))$ contains at least one vertical direction. Clearly, $l_{g} \leq l_{f}$ at every point. Therefore, applying Corollary 12 for $\gamma$ and $g$, at positively many points, the contingent is a non-vertical line. This contradiction proves Theorem 3. It remains to prove Lemma 13

Proof. By Fubini's theorem, there a straight line segment $I \subset[0,1]^{n}$ such that $L_{f}=\infty$ at almost every point of $I$. We denote $I$ as $\gamma_{0}$ and begin our construction of $\gamma$. Fix a small $\epsilon_{0}>0$. At each point $x \in I$ where $L_{f}(x)=\infty$, for an arbitrary small $r$, there is a point $y \in[0,1]^{n}$ with $\|x-y\|<r \epsilon_{0}$ and $|f(x)-f(y)|>r / \epsilon_{0}$. The balls $B(x, r)$ form a Vitali covering, therefore we can choose countably many disjoint balls $B_{i}=B\left(x_{i}, r_{i}\right)$ that cover almost every point $x$ in $I$ with $L_{f}(x)=\infty$. For each $i$, replace the part of $\gamma_{0}$ that is in $B_{i}$, i.e. the line segment $\left[a_{i}, b_{i}\right]:=I \cap B_{i}$, with a polygon from $a_{i}$ to $b_{i}$ that has $x$ and $y$ as its vertices (and possibly it also has some other vertices). We define this new curve as $\gamma_{1}$. We can choose the polygons such that the length of $\gamma_{1}$ is less than $\left(1+\epsilon_{0}\right)|I|$.

Suppose that we have constructed $\gamma_{j}$. In order to construct $\gamma_{j+1}$, we choose a small $\epsilon_{j}$ and perform the same transformation that we applied to $\gamma_{0}$ to each segment of $\gamma_{j}$. However, one obstacle to performing the transformation may be that $L_{f}(x)<\infty$ on a segment of $\gamma_{j}$. To avoid this situation, we can first replace each segment by a polygon, arbitrarily close to the original one, on which $L_{f}=\infty$ at $\mathcal{H}^{1}$ almost every point. Now we can transform each segment of $\gamma_{j}$. It is clear from the geometry that, by choosing the parameters $\epsilon_{j}$ and the balls in the Vitali coverings small enough, in the limit, we obtain a simple curve $\gamma$ of length at most $\prod_{j}\left(1+\epsilon_{j}\right)|I|<\infty$.

The curve $\gamma$ contains countably many exceptional sets of $\mathcal{H}^{1}$ measure zero (namely, those points that are not covered when we apply Vitali's covering theorem), but all other points $x_{0} \in \gamma$ are covered by a ball $B(x, r)$ for some $x \in \gamma$ and an arbitrary small $r$, and there is a $y \in B(x, r) \cap \gamma$ and an arbitrary small $\epsilon$, such that $|f(x)-f(y)|>r / \epsilon$. Then of course $\max \left(\left|f\left(x_{0}\right)-f(x)\right| / r,\left|f\left(x_{0}\right)-f(y)\right| / r\right)>1 / 2 \epsilon$, so indeed $L_{g}\left(x_{0}\right)=\infty$ for $g=\left.f\right|_{\gamma}$.

\section{Higher dimensional SURfaCes AND NON-DifFerentiability}

We devote the rest of this paper to the construction of an $n$-dimensional surface in $\mathbb{R}^{n+1}$ of finite $\mathcal{H}^{n}$ measure, for any $n>1$, that does not have a tangent at any 
of its points (and also have some additional properties). And we show how this construction can be used to prove Theorem 2

Proposition 14. (1) For any $n>1$ there exists a set $S \subset \mathbb{R}^{n+1}$ homeomorphic to $[0,1]^{n}$ that has finite $\mathcal{H}^{n}$ measure, but it does not have a tangent plane at any of its points.

(2) Furthermore, for any $n \geq 1$ and for any gauge function $g$, there exists a set $S \subset \mathbb{R}^{n+1}$ and a homeomorphism $h:[0,1]^{n} \rightarrow S$ that satisfies $l_{h}(x)<\infty$ at every $x \in[0,1]^{n}$ except at the points of a set of zero $\mathcal{H}^{8}$ measure, and $h$ is not differentiable anywhere.

Proposition 14 implies Theorem 2 Indeed, consider the sets

$$
D_{v}:=\left\{x \in[0,1]^{n} \mid v \cdot h \text { is differentiable at } x\right\}
$$

where $v \in \mathbb{R}^{n+1}$ with $\|v\|=1, h$ is the nowhere differentiable homeomorphism in Proposition 14 and $v \cdot h$ is the usual scalar product. If every $D_{v}$ has positive $\mathcal{L}^{n}$ measure, then there are $n+1$ linearly independent directions $v_{i}$ such that $D:=$ $\bigcap_{i} D_{v_{i}} \neq \emptyset$, and then $h$ would be differentiable at the points of $D$. Therefore, there exists $v$ such that $f:=v \cdot h: \mathbb{R}^{n} \rightarrow \mathbb{R}$ is not differentiable at a.e. $x$. And since the scalar product is Lipschitz with Lipschitz constant $1, l_{f}(x) \leq l_{h}(x)$ at every $x$.

Now we turn to the proof of Proposition 14 .

4.1. Construction of $S$. We will construct our surface for $n=2$ for simplicity. An analogous construction will work in any dimension.

Let $S_{0}$ be a (closed) planar unit square in $\mathbb{R}^{3}$. For any $j \geq 0$, we will define a surface $S_{j} \subset \mathbb{R}^{3}$ inductively, and then we will take the limit of these surfaces. Each surface $S_{j}$ will be a union of countably many "edges" (straight line segments) and countably many open "faces" (2-dimensional planar polygonal regions whose boundary line segments are edges of $S_{j}$ ). Naturally, the edges of $S_{0}$ are the four edges of the square, and it has one face, the interior of the square.

Suppose that we already defined $S_{j}$ for some $j \geq 0$. First we decompose the faces of $S_{j}$ into countably many non-overlapping closed squares such that for any two squares $Q, Q^{\prime}$, if the intersection $3 Q \cap 3 Q^{\prime}$ is non-empty, then they are of comparable sizes. We will use the notation $\ell(Q)$ for the sidelength of a square $Q$. We also assume that, for each of our squares $Q, \ell(Q) \ll \operatorname{dist}\left(Q, S_{j} \backslash F\right)$, where $F$ is the face of $S_{j}$ to which $Q$ belongs. In particular, $Q$ has distance $\gg(Q)$ from the edges of $F$.

Next, we modify the surface $S_{j}$ in the interior of the squares $Q$ to obtain $S_{j+1}$. Consider a square $Q$ of our construction, and let $Q^{*}$ be the square centered at the center of $Q$ with sidelength $\ell\left(Q^{*}\right) \ll \ell(Q)$. We place a square pyramid $P$ of angle $\alpha(Q) \ll 1$ onto $Q$, and a square column $C$ of height $\ell(Q)$ onto $Q^{*}$, respectively, as in Figure 1. Then we replace int $Q$ by $\partial(C \cup P) \backslash Q$. We do this inside each of our squares $Q$, with sufficiently small $\ell\left(Q^{*}\right), \alpha(Q)$ that we will specify later. The resulting surface is $S_{j+1}$. 
Figure 1

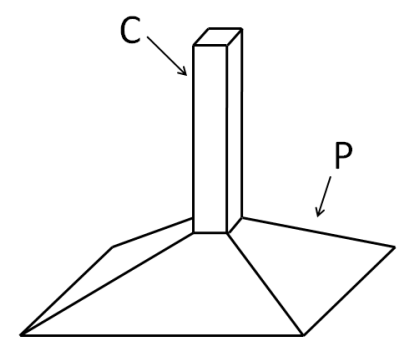

Note that each square $Q$ is replaced by a piecewise affine surface that is in the $\ell(Q)$-neighborhood of $Q$. Using this and the fact that $Q$ has distance $\gg \ell(Q)$ from $S_{j} \backslash F$, it is easy to see that $S_{j+1}$ is homeomorphic to $S_{j}$. Consequently, each $S_{j}$ is homeomorphic to $S_{0}=[0,1]^{2}$. Also, it is clear from the geometry that $S_{j}$ converges to a surface $S$ homeomorphic to $[0,1]^{2}$. Later we will describe a homeomorphism $h_{j}:[0,1]^{2} \rightarrow S_{j}$ explicitly and show that the homeomorphisms $h_{j}$ converge to a homeomorphism $h:[0,1]^{2} \rightarrow S$ that satisfy part (2) of our proposition. But first let us show part (1).

4.2. Finite $\mathcal{H}^{2}$ measure. Let $c>1$ be arbitrary. First note that for each cube $Q$ and its column $C$ and pyramid $P, \mathcal{H}^{2}(\partial(C))<6 \ell\left(Q^{*}\right) \ell(Q)$, and that $\mathcal{H}^{2}(\partial P \backslash Q)-\mathcal{H}^{2}(Q)$ is small if $\alpha(Q)$ is small. Therefore, by choosing $\alpha(Q)$ and $\ell\left(Q^{*}\right)$ small enough (so small that, even $\sum 6 \ell\left(Q^{*}\right) \ell(Q)$ is small where the summation is taken over all squares $Q$ of all steps), we can ensure that $\mathcal{H}^{2}\left(S_{j}\right)<c$ for any $j$.

In order to ensure that not only the surfaces $S_{j}$, but also the limit surface $S$ has finite $\mathcal{H}^{2}$ measure, inductively we define a sequence of positive numbers $\delta_{j}$ tending to zero, and a sequence of open sets $G_{j}$ such that $S_{j} \subset G_{j}$ and $\mathcal{H}_{\delta_{j}}^{2}\left(G_{j}\right)<c$. We can also assume that $G_{0} \supset \mathrm{cl} G_{1} \supset G_{1} \supset \mathrm{cl} G_{2} \supset G_{2} \ldots$ Indeed, if $S_{j}, \delta_{j}, G_{j}$ have been already defined for a given $j$, then in the construction of $S_{j+1}$ we can choose the squares $Q$ so small that their sidelength is less then their distance from $G_{j}^{c}$. Then $S_{j+1} \subset G_{j}$; and we can choose $G_{j+1}, \delta_{j+1}$ with $\mathrm{cl} G_{j+1} \subset G_{j}$ and $\mathcal{H}_{\delta_{j+1}}^{2}\left(G_{j+1}\right)<c$.

Then $S \subset \bigcap_{j} \mathrm{cl}_{j}=\bigcap_{j} G_{j}, \mathcal{H}_{\delta_{j}}^{2}(S) \leq \mathcal{H}_{\delta_{j}}^{2}\left(G_{j}\right)<c$ for any $j$, and consequently, $\mathcal{H}^{2}(S) \leq c$.

Remark 15. We used the assumption $n>1$ in this proof. Indeed, in dimension $n$, the columns have surface area bounded by $2(n+1) \ell\left(Q^{*}\right)^{n-1} \ell(Q)$, which we can make small by choosing $\ell\left(Q^{*}\right)$ small enough, only if $n>1$.

We will not use this assumption anywhere else. We can do the same construction also for $n=1$, it will give us a nowhere differentiable $S$ with $\mathcal{H}^{1}(S)=\infty$. As we have seen, part (1) of Proposition 14 is false for $n=1$. But part (2) will remain true; we will use the construction 4.1 also in dimension $n=1$ when we prove part (2).

4.3. Edges and faces. In our construction, each face of $S_{j}$ is divided into countably many squares, and then each square is replaced by a piecewise affine surface, to obtain $S_{j+1}$. Naturally, we define the faces of $S_{j+1}$ as the faces of these piecewise affine surfaces; and it has two type of edges: firstly, the edges of $S_{j}$ are also edges of $S_{j+1}$, and secondly, the edges of our countably many piecewise affine surfaces. The points of the first type of edges do not belong to the closure of any face of $S_{j+1}$; 
while, at the points of the second type, at least two faces of $S_{j+1}$ meet, in a positive angle.

Note that all edges of $S_{j}$ are preserved by our construction: they are also edges of the surfaces $S_{j+1}, S_{j+2}, \ldots$, and consequently, they belong to the surface $S$.

4.4. No tangents. Next, we show that $S$ does not have a tangent plane at any of its points. First, consider an edge of $S_{j}$ for some $j \geq 1$, and assume that it is not on an edge of $S_{j-1}$. Then two faces of $S_{j}$ meet at this edge in a positive angle, so clearly $S_{j}$ does not have a tangent at the points of this edge. This non existence is preserved during our construction because, on both faces, there is a sequence of squares of $S_{j+1}$ converging to this edge, and the edges of these squares in $S_{j+1}$ will be preserved for every step afterwards. That is, for any point $x$ in any edge, $\operatorname{contg}_{S}(x)$ contains all faces that meet at $x$.

Now consider an $x \in S$ that does not belong to any edge of any $S_{j}$. Then, there is an arbitrary small $r$, such that within the $r$-neighborhood of $x$, there is a $Q \subset S_{j}$ of length comparable to $r$. This follows from our choice of the side lengths of the squares $Q$ : if $Q$ is a square of our construction, then the squares $Q^{\prime}$ used in the next step on the surface of the pyramid $P=P(Q)$ and the column $C=C(Q)$ all have side lengths $\ell\left(Q^{\prime}\right) \ll \ell(Q)$. And in the next steps $\ell\left(Q^{\prime \prime}\right) \ll \ell\left(Q^{\prime}\right)$, etc, therefore during the whole construction, we stay within a neighborhood $\ll \ell(Q)$. This also shows that on an arbitrary small scale $r$, next to $x$ in a distance comparable to $r, S$ "looks like" Figure 1, and not like a plane: it is not within a small angle of any plane. This finishes the proof of part (1).

4.5. Definition of $h$. Let us now define a homeomorphism $[0,1]^{n} \rightarrow S \subset \mathbb{R}^{n+1}$ which will satisfy the requirements in part (2). We will use the construction of the surface $S$ and its properties described in 4.1 and 4.3-4.4.

First, for each $j$, we define a homeomorphism from $S_{0}=[0,1]^{n} \subset[0,1]^{n+1}$ onto $S_{j}$. Let $h_{0}$ be the identity. We define $h_{j}$ as $f_{j} \circ h_{j-1}$ where $f_{j}: S_{j-1} \rightarrow S_{j}$ is a homeomorphism. We let $f_{j}$ to be the identity on $S_{j-1} \cap S_{j}$, and construct $f_{j}$ on each cube $Q \subset S_{j-1}$ individually. Let $\theta$ denote the direction orthogonal to $Q$.

(1) For each $y \in Q \backslash Q^{*}$, let $f_{j}$ be the projection of $y$ onto $\partial P$ in direction $\theta$.

(2) The cube $Q^{*}$ is first mapped by a translation in the direction $\theta$ onto the cube $Q^{\prime}$ defined by $\partial Q^{\prime}=\partial C \cap \partial P$, and then the interior of this cube $Q^{\prime}$ is mapped to $\partial C \backslash \partial P$ by a perspective projection from a point far below the center of $Q^{*}$ in direction $\theta$.

Figure 2

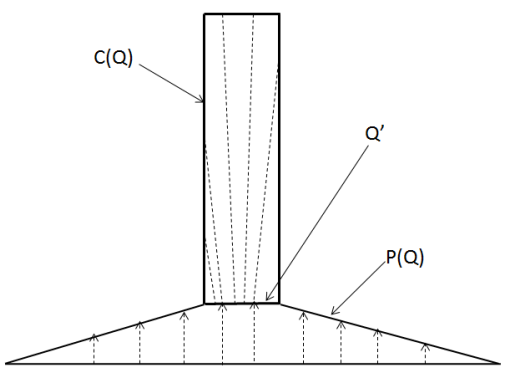

We note that the point used for the perspective projection can be defined far enough from $Q^{*}$ so that the projection increases distances between points. Since the $S_{j}$ are polygonal, $f_{j}$ is piecewise smooth as well as $h_{j}:=f_{j} \circ h_{j-1}$ for $j \geq 1$. 
From section 4.1, we know that $f_{j}(Q)$ lies within an $\ell(Q)$ neighborhood of $Q$. Therefore $c_{j}:=\left\|h_{j}-h_{j-1}\right\|_{\infty} \lesssim \sup l(Q)$, where the supremum is taken over all cubes of $S_{j-1}$. In 4.4 , we have also seen that $c_{j}$ tends to zero (at least) as fast as a geometric sequence, therefore the continuous functions $h_{j}$ converge uniformly to a continuous function $h$. We need to show that $h$ is injective.

First note that, for any face $F$ of $S_{j-1}$ and for any two $x, y \in F,\left\|f_{j}(x)-f_{j}(y)\right\| \gtrsim \| x-$ $y \|$. Now let $x, y \in S_{0}$ be arbitrary, and consider the first $j$ for which $h_{j-1}(x), h_{j-1}(y)$ are not inside the same face of $S_{j-1}$. If $x$ is on an edge of $S_{j-1}$ then $h(x)=h_{j-1}(x)$, and if it is covered by one of our cubes $Q$, then $\left\|h_{j-1}(x)-h_{j}(x)\right\| \lesssim \ell(Q)$ and $\left\|h_{j}(x)-h(x)\right\| \ll \ell(Q)$. We also know that $Q$ has distance $\gg \ell(Q)$ from $S_{j-1} \backslash F$, where $F$ is the face that $Q$ belongs to. In particular, it has distance $\gg \ell(Q)$ from $y$. The same estimates are true for $y$. Putting these estimates together, we can see not only that $h$ is injective, but:

$$
\|x-y\| \lesssim\|h(x)-h(y)\| .
$$

This also shows that $h$ is not differentiable at any point $x$. Indeed, recall that $S$ cannot be well-approximated by a plane at any point,

$$
\|\theta \cdot(h(x)-h(y))\|=o(\|h(x)-h(y)\|)
$$

fails for any direction $\theta$, and of course if $h$ is differentiable at $x$ with derivative $L$ : $\mathbb{R}^{n} \rightarrow \mathbb{R}^{n+1}$, for $\theta \perp \operatorname{Im} L$ we would get $|\theta \cdot(h(x)-h(y))|=o(\|x-y\|) \lesssim o(\|h(x)-h(y)\|)$.

4.6. Lower scaled oscillation. Consider a cube $Q$ on $S_{j}$, its middle cube $Q^{*}$, and the inverse image $Q^{* *}:=h_{j}^{-1}\left(Q^{*}\right)$. Let $E \subset S_{0}$ denote the set of points that belong to a $Q^{* *}$ for infinitely many $j$. Since $\operatorname{diam}\left(Q^{* *}\right) \leq c \operatorname{diam}\left(Q^{*}\right)$ for some absolute constant $c$, by choosing the cubes $Q^{*}$ in our construction small enough (so small that, even $\sum g\left(c \operatorname{diam}\left(Q^{*}\right)\right)<\infty$ where $g$ is the gauge function and the summation is taken over all cubes $Q^{*}$ in all steps), we indeed get $\mathcal{H}^{g}(E)=0$.

Now consider an $x$ in the complement of $E$. Then there is a $j$ such that $h_{k}(x)$ does not belongs to a column in $S_{k}$ for any $k \geq j$. Assume first that $x$ is not mapped to an edge of any $S_{k}$.

In this case we know that $h_{j}$ is smooth around $x$, in particular, it is Lipschitz with some Lipschitz constant $L$ (that depends on $x$ and $j$ ). For $k \geq j$, let $Q_{k}$ denote the cube in $S_{k}$ that $h_{k}(x)$ belongs to. Then $h_{k} \circ h_{j}^{-1}$ is Lipschitz with Lipschitz constant $\alpha:=1+\sum \alpha(Q)$ on $h_{j} \circ h_{k}^{-1}\left(Q_{k}\right)$, where $\alpha(Q)$ is the angle of the pyramid $P(Q)$ as in 4.1 , and the summation is taken over all cubes of all steps so that $\alpha$ does not depend on $k$.

Recall that for any other cube $Q_{k}^{\prime}$ in $S_{k}$, if $3 Q_{k} \cap 3 Q_{k}^{\prime} \neq \emptyset$ then they are of comparable sizes. Therefore, all the cubes that meet $B_{k}:=B\left(h_{k}(x), \ell\left(Q_{k}\right)\right)$ in $S_{k}$ are comparable to $Q_{k}$, therefore the oscillation of $h \circ h_{k}^{-1}$ on $B_{k}$ is comparable to $\ell\left(Q_{k}\right)$, in particular, it is bounded by $C \ell\left(Q_{k}\right)$ for some absolute constant $C$.

Now we are ready to prove that $l_{h}(x)<\infty$. For any $k$, consider the ball $B(x, r)$ for a small $r$. This ball is mapped into the ball $B\left(h_{j}(x), L r\right)$ by $h_{j}$, then into $B\left(h_{k}(x), \alpha L r\right)$ by $h_{k}$. Therefore, with the choice $r:=\ell\left(Q_{k}\right) / \alpha L$, the image of $B(x, r)$ through $h_{k}$ is inside $B_{k}$, and then the image of $h$ is inside the $C \ell\left(Q_{k}\right)$-neighborhood of $B_{k}$. That is,

$$
\sup _{B\left(x, \ell\left(Q_{k}\right) / \alpha L\right)}\|h(x)-h(y)\| \lesssim \ell\left(Q_{k}\right) .
$$

Letting $k \rightarrow \infty$ we obtain $l_{h}(x)<\infty$. 
Finally, when $h_{k}(x)$ is on an edge of $S_{k}$ for some $k$, suppose that $k$ is the first index when this happens. Then it is clear that $l_{h_{k}}(x)<\infty$, and as we proceed with the construction, we only modify our functions on cubes $Q$ whose distance from $h_{k}(x)$ is $\gg \ell(Q)$, therefore in a neighborhood of $x,|h(y)-h(x)|$ is comparable to $\left|h_{k}(y)-h_{k}(x)\right|$.

\section{REFERENCES}

1. G. Alberti, M. Csörnyei, D. Preiss, Paper in preparation.

2. Z.M. Balogh, M. Csörnyei, Scaled-oscillation and regularity, Proc. Am. Math. Soc. 134, No. 9 (2006), 2667-2675.

3. A.S. Besicovitch, On the definition of tangents to sets of infinite linear measure, Proc. Cambridge Philos. Soc. 52 (1956), 20-29.

4. A.S. Besicovitch, Analysis of tangential properties of curves of infinite length, Proc. Cambridge Philos. Soc. 53 (1957), 69-72.

5. K.J. Falconer, Geometry of Fractal Sets, Cambridge Univ. Press, (1985).

6. B. Hanson, Linear dilatation and differentiability of homeomorphisms of $\mathbb{R}^{n}$, Proc. Amer. Math. Soc. 140 (2012), 3541-3547.

7. P. Mattila, Geometry of sets and measures in Euclidean spaces, Cambridge Univ. Press, (1995).

8. F. Roger, Sur la relation entre les propriétés tangentielles et métriques des ensembles cartésiens, C. R. Math. Acad. Sci. Paris. 201 (1935), 871-873.

9. S. Saks, Theory of the Integral, Dover, (1964).

Department of Mathematics, The University of Chicago, 5734 South University Avenue, Chicago, IL 60615, U.S.A. 